5. Chew KL, Cheng B, Lin RTP, Teo JWP. Elizabethkingia anophelis is the dominant Elizabethkingia species found in blood cultures in Singapore. J Clin Microbiol 2018;56:1-3.

6. Wang M, Gao H, Lin N, et al. The antibiotic resistance and pathogenicity of a multidrug-resistant Elizabethkingia anophelis isolate. MicrobiologyOpen 2019;e804.

7. Clinical and Laboratory Standards Institute. Performance Standards for Antimicrobial Susceptibility Testing, 29th edition. Wayne, PA: CLSI; 2013.
8. Adiguzel A, Ozkan H, Baris O, Inan K, Gulluce M, Sahin F. Identification and characterization of thermophilic bacteria isolated from hot springs in Turkey. J Microbiol Meth 2009;79:321-328.

9. Lin JN, Lai CH, Yang CH, Huang YH, Lin HH. Genomic features, phylogenetic relationships, and comparative genomics of Elizabethkingia anophelis strain EM361-97 isolated in Taiwan. Sci Rep 2017;7:8.

\title{
Impact of neutropenia on central venous catheter-related bloodstream infections in patients with hematological malignancies at the time of central venous catheter insertion: A matched-pair analysis
}

\author{
Daniela Tölle $M D^{1}$, Marcus Hentrich $\mathrm{MD}^{2}$, Benedikt W. Pelzer $\mathrm{MD}^{3}$, Pierre Kremer $\mathrm{MD}^{4}$, Sabine Einhell $\mathrm{MD}^{5}$, \\ Sebastian Schulz MD², Boris Böll MD², Jens Panse MD ${ }^{5}$, Martin Schmidt-Hieber MD ${ }^{4}$, Daniel Teschner MD ${ }^{1}$ \\ and Enrico Schalk $M D^{6}$ (i) \\ ${ }^{1}$ Department of Hematology, Medical Oncology, and Pneumology, University Medical Center of the Johannes Gutenberg University Mainz, Mainz, Germany, \\ ${ }^{2}$ Department of Hematology and Oncology, Red Cross Hospital Munich, Munich, Germany, ${ }^{3}$ Department of Internal Medicine I, Center for Integrated Oncology \\ Aachen Bonn Cologne Dusseldorf, University Hospital of Cologne, Cologne, Germany, ${ }^{4}$ Clinic of Hematology and Oncology, Carl-Thiem Hospital Cottbus, Cottbus, \\ Germany, ${ }^{5}$ Department of Oncology, Hematology, Hemostaseology and Stem Cell Transplantation, Medical Faculty, University Hospital RWTH Aachen, Aachen, \\ Germany and ${ }^{6}$ Department of Hematology and Oncology, Medical Center, Otto-von-Guericke University Magdeburg, Magdeburg, Germany
}

To the Editor-Central venous catheter (CVC)-related bloodstream infections (CRBSIs) are potentially preventable complications associated with high morbidity, especially in patients with hematological malignancies. ${ }^{1}$ Neutropenia is one of the most important risk factors for CRBSI ${ }^{1,2}$ because of insufficient immune control of the patient's microbial flora or pathogens when absolute neutrophil counts (ANC) are $<500 / \mu \mathrm{L}^{3,4}$ Neutropenia as a risk factor for CRBSI is only well defined for the time of CRBSI onset. ${ }^{5,6}$ So far, no reliable data are available that address the impact of neutropenia on CRBSI at the time of CVC insertion. This impact is of special interest for hematology patients because CVCs are often inserted during neutropenia either due to the underlying malignancy, like acute myeloid leukemia (AML), or after application of chemotherapy. In addition, CVC reinsertions after CVC removal due to CRBSI are also common, especially during longlasting neutropenia, for example, after remission-inducing chemotherapy in AML patients or after conditioning therapies before hematopoietic stem-cell transplantation (HSCT). Therefore, we aimed to investigate the impact of neutropenia on subsequent CRBSI at the time of insertion of short-term, nontunneled CVCs in adult patients with hematological malignancies.

We analyzed data from the prospective multicenter SECRECY study (German Clinical Trial Register, no. DRKS00006551), a CRBSI registry conducted in 6 German hematology and oncology centers, including the aforementioned group of high-risk patients

Author for correspondence: Enrico Schalk, MD, Otto-von-Guericke University Magdeburg Medical Center, Department of Hematology and Oncology, Leipziger Str 44, D-39120 Magdeburg, Germany. E-mail: enrico.schalk@med.ovgu.de

Cite this article: Tölle D, et al. (2019). Impact of neutropenia on central venous catheter-related bloodstream infections in patients with hematological malignancies at the time of central venous catheter insertion: A matched-pair analysis. Infection Control \& Hospital Epidemiology, 40: 1204-1206, https://doi.org/10.1017/ice.2019.224 receiving AML induction or HSCT. Inclusion criteria encompassed short-term, nontunneled jugular and subclavian vein CVCs with $\geq 1$ day in situ, and CRBSI was classified according to the 2012 Infectious Diseases Working Party (AGIHO) of the German Society for Hematology and Medical Oncology (DGHO) CRBSI definition. ${ }^{2}$ Only CRBSIs classified as definite or probable were considered. Hence, we identified 2,368 patients with a total follow-up of 37,932 CVC days. In 2,158 cases $(91.1 \%)$, the underlying diseases were hematological malignancies. Among them, we identified 406 cases $(17.1 \%)$ with neutropenia defined as ANC $<500 / \mu \mathrm{L}$ or white blood cell counts $<1,000 / \mu \mathrm{L}$ at the time of CVC insertion. Of 406 cases, 400 could be matched for age, sex, underlying disease, site of CVC insertion, use of chlorhexidine-coated CVC dressings, use of chlorhexidine-, antimicrobial- or silver sulfadiazine-coated CVCs, and complications during CVC insertion.

The median age of the patients was 59 years, and $60.3 \%$ were male (Table 1). Approximately $75 \%$ of CVCs were inserted in patients suffering from acute leukemia, and almost all CVCs were placed in the jugular vein (388 of 400, 97.0\%). In a minority of patients, coated CVCs were inserted (23 of $400,5.8 \%$ ), whereas in half of the cases chlorhexidine-coated CVC dressings were used (196 of 400, 49.0\%). Known high-risk CVC features (ie, male sex, complicated CVC insertion, diagnosis of AML, multiple myeloma, or non-Hodgkin lymphoma ${ }^{7}$ ) were present in half of the patients (203 of 400, 50.8\%). The median CVC time in situ was nonsignificantly shorter in CVCs inserted during neutropenia compared to CVCs in nonneutropenic patients (14 vs 18 days; $P=.39$ ). We found no differences in CRBSI rates comparing neutropenic to nonneutropenic patients (45 of 400 [11.3\%] vs 50 of 400 [12.5\%]; $P=.66)$. However, median time to CRBSI diagnosis was shorter in patients who received the CVC in neutropenia 
Table 1. Demographics and Characteristics of CVCs and CRBSI Patients

\begin{tabular}{|c|c|c|c|}
\hline Parameter & $\begin{array}{l}\text { Neutropenic at CVC Insertion } \\
\qquad(n=400)\end{array}$ & $\begin{array}{l}\text { Nonneutropenic at CVC Insertion } \\
\qquad(n=400)\end{array}$ & $P$ Value \\
\hline Median age, years (IQR) & $59(51-66)$ & $59(47-67)$ & .86 \\
\hline Males, n/N (\%) & $241 / 400(60.3)$ & $241 / 400(60.3)$ & 1.0 \\
\hline Underlying diseases, $\mathrm{n} / \mathrm{N}(\%)$ & & & 1.0 \\
\hline Acute myeloid leukemia & $263 / 400(65.8)$ & $263 / 400(65.8)$ & \\
\hline Acute lymphoblastic leukemia & $29 / 400(7.3)$ & $29 / 400(7.3)$ & \\
\hline Multiple myeloma & $28 / 400(7.0)$ & $28 / 400(7.0)$ & \\
\hline Non-Hodgkin lymphoma & $25 / 400(6.3)$ & $25 / 400(6.3)$ & \\
\hline Hodgkin lymphoma & $9 / 400(2.3)$ & $9 / 400(2.3)$ & \\
\hline Other & $46 / 400(11.5)$ & $46 / 400(11.5)$ & \\
\hline CHG-coated CVC dressing, n/N (\%) & $196 / 400(49.0)$ & $196 / 400(49.0)$ & 1.0 \\
\hline Coated CVC, n/N (\%) & $23 / 400(5.8)$ & $23 / 400(5.8)$ & 1.0 \\
\hline Jugular vein CVC, n/N (\%) & $388 / 400(97.0)$ & $388 / 400(97.0)$ & 1.0 \\
\hline Complicated CVC insertion, n/N (\%) & $52 / 400(13.0)$ & $52 / 400(13.0)$ & 1.0 \\
\hline High-risk CVCa, $\mathrm{n} / \mathrm{N}(\%)$ & $203 / 400(50.8)$ & $203 / 400(50.8)$ & 1.0 \\
\hline CVC days, in total & 6,614 & 7,240 & \\
\hline CVC days, median (IQR) & $14(9-24)$ & $18(10-25)$ & .39 \\
\hline Median time to CRBSI onset, d (IQR) & $10(7-17)$ & $15(11-19)$ & .002 \\
\hline CRBSI, n/N (\%) & 45/400 (11.3) & $50 / 400(12.5)$ & .66 \\
\hline Definitive, n/N (\%) & $24 / 45(53.3)$ & $27 / 50(54.0)$ & 1.0 \\
\hline Probable, n/N (\%) & $21 / 45(46.7)$ & $23 / 50(46.0)$ & \\
\hline CRBSI incidence, $x / 1,000$ CVC days & 7.1 & 7.1 & .96 \\
\hline CRBSI-14, \% (95\% CI) & $11.1(7.4-14.8)$ & $7.8(4.7-10.9)$ & .07 \\
\hline CRBSI-21, \% (95\% CI) & $15.4(11.1-19.7)$ & $15.7(11.2-20.2)$ & .71 \\
\hline Causative pathogens, n/N (\%) & & & .26 \\
\hline Coagulase-negative staphylococci & $39 / 45(86.7)$ & $38 / 50(76.0)$ & \\
\hline Other Gram positives & $4 / 45(8.9)$ & $2 / 50(4.0)$ & \\
\hline Enterobacteriaceae & $1 / 45(2.2)$ & $2 / 50(4.0)$ & \\
\hline Other Gram negatives & $1 / 45(2.2)$ & $4 / 50(8.0)$ & \\
\hline Candida spp & $0 / 45(0)$ & $2 / 50(4.0)$ & \\
\hline Multibacterial & $0 / 45(0)$ & $2 / 50(4.0)$ & \\
\hline
\end{tabular}

compared to non-neutropenic controls (10 vs 15 days; $P=.002$ ). Generally, definitive CRBSIs were more often diagnosed than probable CRBSIs without differences between the neutropenic and the nonneutropenic patient groups. Furthermore, there were no differences in the CRBSI incidence (7.1 vs 7.1 per 1,000 CVC days; $P=.96$ ) or in the CRBSI probability over the time on day 14 (hazard ratio [HR], 1.64; 95\% confidence interval [CI], $0.96-2.49 ; P=.07)$ or on day 21 (HR, 1.09; 95\% CI, 0.70-1.70; $P=.71)$. Predominantly, coagulase-negative staphylococci $(\sim 80 \%)$ were documented as the causative pathogens for CRBSI without differences in both groups. Approximately half of the CVCs inserted during neutropenia were removed during neutropenia (190 of 400, 47.5\%), while this was only the case in one-third of the CVCs inserted in nonneutropenic patients (134 of 400,
33.5\%; $P<.001)$. At the time of CRBSI diagnosis, we found no significant impact of neutropenia during CVC insertion (38 of 45 [84.4\%] vs 38 of 50 [76.0\%]; $P=.44$ ). We found no positive impact for chlorhexidine-coated CVC dressings compared to standard dressings (HR, 0.92; 95\% CI, 0.48-1.75; $P=.79$ ) regarding CRBSI probability on day 21 for patients with neutropenia at the time of CVC insertion. The same could be shown for patients without neutropenia at the time of CVC insertion (HR, 0.75; 95\% CI, 0.41-1.39; $P=.36$ ).

In multivariate Cox regression analysis, persisting neutropenia from CVC insertion until CRBSI diagnosis (HR, 1.73; 95\% CI, $1.09-2.73 ; P=.019)$ and neutropenia at the time of CRBSI diagnosis (HR, 2.57; 95\% CI, 1.33-4.95; $P=.005$ ) were independent risk factors for CRBSI, but neutropenia at the time of CVC insertion $(P=.89)$ or 
resolution of neutropenia from CVC insertion until CRBSI diagnosis $(P=.93)$ had no impact. Furthermore, use of chlorhexidine-coated CVC dressings $(P=.19)$ or coated CVCs $(P=.91)$ did not influence CRBSI probability in our multivariate analysis.

Here, we provide data on the potential impact of neutropenia at the time of insertion of short-term, nontunneled CVCs in a large cohort of patients at high risk for CRBSI. According to our registry data, CVC insertion during neutropenia is safe and feasible and not associated with an increased CRBSI risk but with an earlier CRBSI onset. Notably, use of chlorhexidine-coated CVC dressings or coated CVCs does not significantly prevent CRBSI in patients with hematological malignancies at high risk for CRBSI. However, presence of neutropenia at the time of CRBSI diagnosis is still associated with higher morbidity, which highlights the importance of careful CVC handling and management in this vulnerable patient cohort.

Financial support. No financial support was provided relevant to this article.

Conflicts of interest. All authors report no conflicts of interest relevant to this article.

\section{References}

1. Zakhour R, Chaftari AM, Raad II. Catheter-related infections in patients with haematological malignancies: novel preventive and therapeutic strategies. Lancet Infect Dis 2016;16:e241-e250.
2. Hentrich M, Schalk E, Schmidt-Hieber M, et al. Central venous catheterrelated infections in hematology and oncology: 2012 updated guidelines on diagnosis, management and prevention by the Infectious Diseases Working Party of the German Society of Hematology and Medical Oncology. Ann Oncol 2014;25:936-947.

3. Bodey GP, Buckley M, Sathe YS, Freireich EJ. Quantitative relationships between circulating leukocytes and infection in patients with acute leukemia. Ann Intern Med 1966;64:328-340.

4. Holland SM, Gallin JI. Disorders of granulocytes and monocytes. In: Kasper DL et al., eds. Harrison's Principles of Internal Medicine, 16th ed. New York: McGraw-Hill; 2005: 349-357.

5. Howell PB, Walters PE, Donowitz GR, Farr BM. Risk factors for infection of adult patients with cancer who have tunneled central venous catheters. Cancer 1995;75:1367-1375.

6. Wisplinghoff H, Seifert H, Wenzel RP, Edmond MB. Current trends in the epidemiology of nosocomial bloodstream infections in patients with hematological malignancies and solid neoplasms in hospitals in the United States. Clin Infect Dis 2003;36:1103-1110.

7. Schalk E, Toelle D, Schulz S, et al. Identifying haematological cancer patients with high risk for central venous catheter (CVC)-related bloodstream infections at the time point of CVC insertion. 29th European Congress of Clinical Microbiology and Infectious Diseases, April 13-16, 2019, Amsterdam, The Netherlands (abstract P2556). https://www.escmid.org/ escmid_publications/escmid_elibrary. Published 2019. Accessed May 17, 2019.

\title{
Need for heightening awareness of congenital rubella syndrome in the United States
}

\author{
Aditi Mahesh Gupta $\mathrm{MD}^{1}$ and Ritu Cheema $\mathrm{MD}^{2}$ (1) \\ ${ }^{1}$ Department of Pediatrics, University of California, Davis School of Medicine, Sacramento, California and ${ }^{2}$ Division of Pediatric Infectious Diseases, Department \\ of Pediatrics, University of California, Davis School of Medicine, Sacramento, California
}

To the Editor - Congenital rubella syndrome (CRS) is seen more commonly in developing countries due to the lack of rubella vaccine as a part of their national immunization programs, thus leading to the continued disease burden in this part of the world. CRS incidence had considerably decreased in the United States since the introduction of rubella vaccine in 1969 and was considered eliminated from the United States in 2004, although occasional cases (no native cases, however) have been reported in literature since then. ${ }^{1}$ Given the increased travel into those developing countries, increasing immigration of susceptible population (unimmunized due to lack of resources) to the United States, and decreasing vaccination rates in the United States (unimmunized due to exemptions), the incidence of rubella may be on the rise. ${ }^{2}$ Physicians, especially of the younger generation, may have rarely seen a case of congenital rubella syndrome due to previous effective successful immunization program. Furthermore, CRS identification can be challenging because initial symptoms may be consistent with other congenital

Author for correspondence: Ritu Cheema, 2516 Stockton Boulevard, Sacramento, CA 95817. E-mail: ritcheema@ucdavis.edu

Cite this article: Gupta AM and Cheema R. (2019). Need for heightening awareness of congenital rubella syndrome in the United States. Infection Control \& Hospital Epidemiology, 40: 1206-1207, https://doi.org/10.1017/ice.2019.214 infections, as well and findings of sensorineural hearing loss or developmental delay may be identified only later in life.

We discuss here a case highlighting 3 important points: (1) CRS should still be on the differential of congenital infections work up in developed countries; (2) early recognition for CRS patients assists in early intervention and can have profound impacts on neurodevelopmental outcomes; and (3) CRS could have significant infection control implications postdiagnosis, and appropriate isolation precautions need to be followed to limit the spread of the virus.

A late preterm infant was born to a 27 -year-old mother who had recently immigrated from Afghanistan. On initial exam, the infant was noted to have a grade 4/6 systolic heart murmur and "blueberry muffin lesions" on her face and trunk, which faded quickly after birth. She had bilateral cataracts (later requiring repair and lens replacement) and hypotonia. Because of the rash, echocardiogram findings of patent ductus arteriosus, persistent pulmonic stenosis, and thrombocytopenia, congenital rubella were considered in the differential diagnosis. Long-bone X-rays reported "celery stalk metaphysis," and she failed a hearing test as well. Serology returned positive for rubella IgM, confirmed with polymerase chain reaction (PCR) from cerebrospinal, oral, and urine specimens.

Apparently, the mother had received the live rubella vaccine in preparation for her immigration, early during her pregnancy 